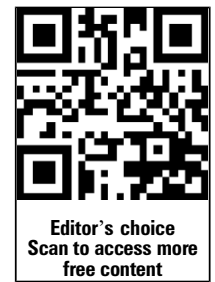

${ }^{1}$ Division of Cardiac Surgery, The Johns Hopkins Hospital, Baltimore, Maryland, USA ${ }^{2}$ Division of Cardiac Surgery, University of Ottawa Heart Institute, Ottawa, Ontario, Canada

${ }^{3}$ Department of Epidemiology and Community Medicine, University of Ottawa Heart Institute, Ottawa, Ontario, Canada

Correspondence to Dr Marc Ruel, Division of Cardiac Surgery, University of Ottawa Heart Institute, 40 Ruskin Street, Ottawa, Ontario, Canada K1Y 4W7: mruel@ottawaheart.ca

Received 16 October 2013 Revised 1 April 2014 Accepted 2 April 2014 Published Online First 19 May 2014

\title{
The impact of prosthesis-patient mismatch after aortic valve replacement varies according to age at operation
}

\author{
Joel Price, ${ }^{1}$ Hadi Toeg $_{1}{ }^{2}$ Buu-Khanh Lam, ${ }^{2}$ Harry Lapierre, ${ }^{2}$ Thierry G Mesana, ${ }^{2}$ \\ Marc Ruel ${ }^{2,3}$
}

\begin{abstract}
Objectives Age may modify the impact of prosthesispatient mismatch (PPM) on outcomes after aortic valve replacement (AVR), as physical functioning decreases with age, and comorbidities become more prevalent. We hypothesised that the consequences of PPM in patients 70 years old or older may be less important than in younger patients.

Methods In total, 707 aortic stenosis patients were followed for a maximum of 17.5 years after AVR. PPM was defined as an in vivo indexed effective orifice area $\leq 0.85 \mathrm{~cm}^{2} / \mathrm{m}^{2}$, and severe PPM as $\leq 0.65 \mathrm{~cm}^{2} / \mathrm{m}^{2}$.

Results In patients less than 70 years of age with normal LV function, the presence of PPM did not significantly alter survival. However, in patients under 70 with LV dysfunction, PPM was associated with decreased survival (HR 2.2; $p=0.046)$. In patients aged 70 years of age or older, PPM had no effect on survival, regardless of LV function. Similarly, PPM was predictive of postoperative congestive heart failure (CHF) in patients under 70 with LV dysfunction (HR 3.6; $p=0.046$ ) but not in older patients. Similar results were observed for the composite endpoint of death or CHF. Postoperative LV mass regression was impaired by increased age $(p=0.019)$, and by PPM in patients aged 70 years of age or older with LV dysfunction (by $28.8 \mathrm{~g} / \mathrm{m}^{2}$; $\mathrm{p}=0.026$ ).
\end{abstract}

Conclusions The impact of PPM on outcomes after AVR depends on age at operation. PPM in patients under age 70 years with LV dysfunction is associated with decreased survival and lower freedom from CHF. In patients 70 years of age or older, PPM does not impact mortality or symptoms, but impairs LV mass regression beyond that explained by age alone.

\section{INTRODUCTION}

Aortic valve replacement (AVR) for aortic stenosis (AS) increases survival, relieves congestive heart failure (CHF), and enables regression of LV hypertrophy. ${ }^{1}$ Prosthesis-patient mismatch (PPM) refers to a situation where the orifice size of the prosthetic valve is too small relative to patient body size. The presence of PPM is associated with elevated transprosthetic gradients, ${ }^{2}{ }^{3}$ and may limit the clinical benefits after AVR.

The impact of PPM on outcomes after AVR continues to be debated. Some maintain it has no effect on mortality. ${ }^{4}$ Others suggest that PPM is associated with poorer outcomes in important subgroups. Some demonstrate that PPM increases mortality, ${ }^{6-11}$ reduces LV mass regression ${ }^{12}{ }^{13}$ and increases incidence of postoperative $\mathrm{CHF}^{8}{ }^{14}$ Previous data from our group indicated that PPM is particularly detrimental to patients with abnormal left ventricular function. ${ }^{6} 15$ These patients experience reduced survival, less LV mass regression, and decreased freedom from CHF.

Age may be an important modifier of the relationship between PPM and outcomes. Elderly patients generally have lower baseline physical functioning and competing mortality risks. These factors may limit their propensity to suffer deleterious outcomes as a result of PPM. The purpose of our study was to examine the effect of PPM on long-term survival, and the related outcomes of freedom from $\mathrm{CHF}$ and $\mathrm{LV}$ mass regression, according to age at the time of operation. We hypothesised that the implications of PPM in AS patients 70 years old or older at the time of operation may be less important than in younger patients. Additionally, we examined the possible interaction between age, preoperative LV function and PPM on outcomes after AVR.

\section{METHODS}

\section{Patient cohort and follow-up}

The patient cohort consisted of 707 adult patients who underwent first-time AVR at the University of Ottawa Heart Institute between 1990 and 2003. The total follow-up was 6174 patient-years, with a median duration of 7.3 (IQR 6.7) years (60 days to 17.5 years). All patients underwent operation for AS (mean gradient $>30 \mathrm{~mm} \mathrm{Hg}$ and $\leq 2+$ insufficiency). Patients who survived the operation, and followed-up at least once were included in the analyses. Patients who underwent concomitant mitral valve surgery were excluded.

The outcomes assessed included overall survival, postoperative $\mathrm{CHF}$, the combined endpoint of allcause mortality or CHF symptoms, and indexed LV mass (LVMI) regression. Postoperative CHF was defined as the composite endpoint of New York Heart Association functional class III or IV symptoms for more than four consecutive weeks, or death where the primary or contributing diagnosis was $\mathrm{CHF}^{7}{ }^{14}$

The in vivo effective orifice area (EOA) for each prosthesis type and size was obtained from the literature of patients with normally functioning prostheses. ${ }^{14} 16$ The indexed EOA was obtained by dividing the in vivo EOA by the patient's body 
surface area (BSA) at the time of operation. PPM was defined as an indexed EOA of $\leq 0.85 \mathrm{~cm}^{2} / \mathrm{m}^{2}$ of BSA; severe PPM was defined as an indexed EOA of $\leq 0.65 \mathrm{~cm}^{2} / \mathrm{m}^{2}$.

Patients underwent postoperative echocardiographic examinations on a biannual basis or as clinically indicated. Modified Bernoulli equations were used to derive peak and mean transvalvular or transprosthesis pressure gradients. Left ventricular end-diastolic and end-systolic diameters, septum and posterior wall thicknesses, and left atrial anteroposterior diameters were measured from the M-mode recordings according to the recommendations of the American Society of Echocardiography. ${ }^{17}$ Left ventricular mass was calculated using the modified formula of the American Society of Echocardiography. ${ }^{18}$ Echocardiographic LV mass changes were derived from the lowest postoperative LV mass minus the preoperative LV mass. These were indexed to preoperative BSA. To assess the effect of PPM according to preoperative left ventricular function, patients were divided into those with normal (LVEF $\geq 50 \%$ ) and impaired LVEF.

\section{Statistical analyses}

Data were analysed using SAS statistical software (SAS Institute, Cary, North Carolina, USA). Continuous data are presented as median (IQR). Differences between patients with and without PPM were analysed, stratified by age by using a $\chi^{2}$ test or Fisher's Exact test for categorical variables, and a Wilcoxon rank-sum test for continuous variables.

Cox proportional hazards modelling was used to examine the independent influence of age and LV function on PPM and survival and/or CHF. A Cox model was constructed for each of the four subsets of age and LV function. Age was dichotomised at 70 years $(<70$ and $\geq 70)$ old and LV function was dichotomised into normal and impaired. All variables listed in table 1 were tested for inclusion in each Cox model. No automated model selection procedure was performed. The number of covariates in the models was restricted to avoid overfitting. The adjustment variables included in each of the final models are listed in table 2. Testing of the proportional hazard assumption was performed with the addition of individual time-varying covariates for each variable in the final model. A similar stratified analysis was used for LVMI with linear regression analysis performed within each of the four subsets.

A number of prespecified sensitivity analyses were performed. These included substitution of a severe PPM threshold into the Cox model for relevant subgroups. In this analysis, severe was compared with no PPM. Additionally, varying age thresholds were substituted into the models for relevant subgroups of LV function.

\section{RESULTS}

\section{Patient characteristics}

Table 1 summarises the patient and operative characteristics of the cohort according to PPM, separately for each age group. The incidence of PPM in patients 70 years old or older was $68 \%(189 / 279)$ compared to $26 \%(110 / 428)$ in patients less than 70 years old $(p=0.0001)$. Patients with PPM were more often female, and PPM was more often associated with use of a bioprosthetic valve. The incidence of severe PPM in patients 70 years old or older was $16.5 \%(46 / 279)$, compared to $4.5 \%$ $(20 / 428)$ in patients less than 70 years old $(\mathrm{p}<0.0001)$.

Table 3 displays the baseline echocardiographic parameters of the cohort. In patients less than 70 years of age, those with no mismatch had larger end-diastolic and end-systolic dimensions than those with mismatch. Patients with mismatch had lower LV mass index than those without. There were no other significant differences in any of the subgroups.

\section{Survival}

Among the 707 patients, there were 188 deaths over the course of follow-up. Age at the time of surgery was a significant

Table 1 Patient characteristics

\begin{tabular}{|c|c|c|c|c|c|c|}
\hline & \multicolumn{2}{|l|}{ Age $<70$ yrs } & \multirow[b]{2}{*}{ p Value } & \multicolumn{2}{|l|}{ Age $\geq 70$ yrs } & \multirow[b]{2}{*}{ p Value } \\
\hline & No mismatch $(n=318)$ & Mismatch $(n=110)$ & & No mismatch $(n=90)$ & Mismatch $(n=189)$ & \\
\hline \multicolumn{7}{|c|}{ Preoperative characteristics (\%) } \\
\hline Diabetes & $14(4.4)$ & $8(7.3)$ & 0.2 & $2(2.2)$ & $10(5.3)$ & 0.3 \\
\hline Hypertension & $166(52.2)$ & $62(56.4)$ & 0.5 & $58(64.4)$ & $112(59.3)$ & 0.4 \\
\hline Prior myocardial infarction & $2(0.63)$ & $1(0.91)$ & 1.0 & 0 & 0 & - \\
\hline $\mathrm{BMI} \geq 30$ & $48(40.0)$ & $15(34.1)$ & 0.7 & $6(17.1)$ & $8(12.7)$ & 0.5 \\
\hline Male sex & $231(72.6)$ & $64(58.2)$ & 0.005 & $61(67.8)$ & $105(55.6 \%$ & 0.06 \\
\hline CABG & $79(24.8)$ & $38(34.6)$ & 0.05 & $41(45.6)$ & $85(45.0)$ & 0.9 \\
\hline Atrial fibrillation & $11(3.5)$ & $0(0.0)$ & 0.05 & $1(1.1)$ & $10(5.3)$ & 0.1 \\
\hline NYHA class & & & 0.5 & & & 0.7 \\
\hline 1 & $85(26.8)$ & $24(21.8)$ & & $18(20.0)$ & $50(26.5)$ & \\
\hline II & $104(32.7)$ & $33(30.0)$ & & $21(23.3)$ & $39(20.6)$ & \\
\hline III & $85(26.8)$ & $36(32.7)$ & & $36(40.0)$ & $73(38.6)$ & \\
\hline IV & $44(13.8)$ & $17(15.5)$ & & $15(16.7)$ & $27(14.3)$ & \\
\hline LVEF $<50 \%$ & $106(33.3)$ & $28(25.5)$ & 0.1 & $36(40.0)$ & $56(29.6)$ & 0.09 \\
\hline Emergency procedure & $9(2.8)$ & $2(1.8)$ & 0.6 & $2(2.3)$ & $5(2.7)$ & 0.8 \\
\hline \multicolumn{7}{|l|}{ Operative characteristics (\%) } \\
\hline Bioprosthetic valve & $53(16.7)$ & $69(62.7)$ & $<.0001$ & $38(42.2)$ & $176(93.1)$ & $<.0001$ \\
\hline Aortic root enlargement ${ }^{*}$ & $34(10.7)$ & $16(14.6)$ & 0.3 & $10(3.6)$ & $14(7.4)$ & 0.3 \\
\hline
\end{tabular}


Table 2 Variables included in final multivariable models

\begin{tabular}{|c|c|c|}
\hline $\begin{array}{l}\text { Outcome } \\
\text { variable }\end{array}$ & Model types & Adjustment variables \\
\hline Survival & Cox & Atrial fibrillation, concomitant $\mathrm{CABG}$ \\
\hline CHF & Cox & Preoperative CHF class \\
\hline Survival/CHF & Cox & $\begin{array}{l}\text { Preoperative CHF class, atrial fibrillation, } \\
\text { concomitant CABG }\end{array}$ \\
\hline LVMI & Linear regression & $\begin{array}{l}\text { Preoperative CHF class, atrial fibrillation, } \\
\text { concomitant } C A B G\end{array}$ \\
\hline
\end{tabular}

independent predictor of decreased long-term survival (HR 1.04 per 1 year increase; 95\% CI 1.02 to 1.06 ; $\mathrm{p}<0.0001$ ).

Figure 1 displays the effect of PPM on survival after AVR stratified by age and LV function. In the subset of patients under 70 years of age with normal LV function, there was no significant difference in long-term survival (HR 1.6; 95\% CI 0.9 to $2.7 ; p=0.10)$. However, in the subset of younger patients with LV dysfunction, PPM was associated with decreased long-term survival (HR 2.2; 95\% CI 1.01 to $4.6 ; \mathrm{p}=0.046$ ). In patients 70 years old and older, PPM had no significant effect on survival, regardless of LV function.

To examine the robustness of these findings, several sensitivity analyses were performed. The effect of age was further explored by substituting different age thresholds into the multivariate equations. For patients with normal preoperative LV function, PPM did not adversely affect survival even in the subset of patients under age 65 years, or those under age 60 years. To examine the role of PPM severity on consequences of mismatch, a PPM threshold of $0.65 \mathrm{~cm}^{2} / \mathrm{m}^{2}$ was substituted into a model for the subgroup of young patients with normal LV function and for both subgroups of older patients. No group experienced a significant reduction in survival as a result of severe PPM compared with no PPM.

\section{CHF or CHF death}

Figure 2 displays the effect of PPM on freedom from CHF after AVR, stratified by age and LV function. In the subset of patients under 70 years of age with normal LV function, the presence of PPM did not affect significantly the freedom from CHF (HR $1.5 ; 95 \%$ CI 0.6 to $3.9 ; p=0.41$ ). However, in patients less than 70 years of age with LV dysfunction, PPM was associated with increased CHF (HR 3.6; 95\% CI 1.02 to 12.4; p=0.046). In patients 70 years old and older, PPM had no significant independent effect on CHF, regardless of LV function.
In patients with normal preoperative LV function, PPM did not adversely affect freedom from CHF even in patients under age 65 years and those under age 60 years. A PPM threshold of $0.65 \mathrm{~cm}^{2} / \mathrm{m}^{2}$ was substituted into a model for the subgroup of young patients with normal LV function and for both subgroups of older patients. No group experienced a significant increase in $\mathrm{CHF}$ as a result of severe PPM.

\section{Death or CHF}

Figure 3 displays the effect of PPM on freedom from the composite endpoint of death or CHF after AVR, stratified by age and LV function. In patients under 70 years of age with normal LV function, the presence of PPM did not affect significantly freedom from the combined endpoint (HR 1.6; 95\% CI 0.91 to 2.7; $\mathrm{p}=0.11$ ). However, in patients less than 70 years of age with LV dysfunction, PPM was associated with increased death or CHF (HR 2.1; 95\% CI 1.0 to $4.5 ; \mathrm{p}=0.049$ ). In patients 70 years old and older, PPM had no significant independent effect on death or CHF, regardless of LV function.

In patients with normal preoperative LV function, PPM did not adversely affect freedom from the composite endpoint even in patients under age 65 years and those under age 60 years. A PPM threshold of $0.65 \mathrm{~cm}^{2} / \mathrm{m}^{2}$ was substituted into a model for the subgroup of young patients with normal LV function and for both subgroups of older patients. No group experienced a significant increase in death or CHF as a result of severe PPM.

\section{LV mass}

Figure 4 depicts the observed maximum LVMI regression in the various subgroups. Maximum LVMI regression was observed at a mean of $1.6 \pm 1.5$ years in patients under age 70 years, and at $1.3 \pm 1.0$ years in patients 70 years old or older. Increasing age was found to be an independent predictor of decreased LV mass regression. After adjusting for confounders, patients 70 years old or older experienced a mean reduction of LVMI regression of $9.8 \mathrm{~g} / \mathrm{m}^{2}(95 \%$ CI 1.6 to $18.0 ; \mathrm{p}=0.019)$ compared with patients aged less than 70 years of age. This remains statistically significant when age was analysed as a continuous variable. Additionally, a normal LV was independently predictive of reduced LVMI regression. PPM did not predict LVMI regression.

In patients under the age of 70 years of age, there was no significant difference in LVMI regression as a result of PPM regardless of LV function preoperatively. In patients over 70 years of age with normal LV function, PPM did not significantly affect LVMI regression $\left(-9.5 \mathrm{~g} / \mathrm{m}^{2} ; 95 \%\right.$ CI -22.8 to $\left.3.3 ; \mathrm{p}=0.14\right)$. However, in patients over 70 years of age in whom the LV was impaired, LVMI regression was reduced in the presence of PPM

Table 3 Preoperative echocardiographic parameters

\begin{tabular}{|c|c|c|c|c|c|c|}
\hline & \multicolumn{2}{|l|}{ Age $<70$ yrs } & \multirow[b]{2}{*}{ p Value } & \multicolumn{2}{|l|}{ Age $\geq 70$ yrs } & \multirow[b]{2}{*}{$p$ Value } \\
\hline & No mismatch $(n=318)$ & Mismatch $(n=110)$ & & No mismatch $(n=90)$ & Mismatch $(n=189)$ & \\
\hline EDD $(\mathrm{cm})$ & $5.6(1.4)$ & $5.2(1.25)$ & 0.001 & $5.2(1.0)$ & $5.1(1.0)$ & 0.23 \\
\hline $\mathrm{ESD}(\mathrm{cm})$ & $3.5(1.5)$ & $3.2(1.3)$ & 0.015 & $3.4(1.4)$ & $3.1(1.1)$ & 0.23 \\
\hline IVS (cm) & $1.1(0.3)$ & $1.1(0.3)$ & 0.37 & $1.1(0.2)$ & $1.1(0.2)$ & 1.0 \\
\hline LVM (g) & $246.7(102.7)$ & $226.1(144.8)$ & 0.28 & $232.2(76.4)$ & $223.5(88.1)$ & 0.45 \\
\hline LVMI $\left(\mathrm{g} / \mathrm{m}^{2}\right)$ & $134.8(57.1)$ & $125.5(70.8)$ & 0.033 & $135.6(50.1)$ & $112.6(48.8)$ & 0.0001 \\
\hline FS (\%) & 36.7 (14.4) & $39.0(14.7)$ & 0.37 & 34.7 (15.8) & $38.1(15.2)$ & 0.30 \\
\hline
\end{tabular}



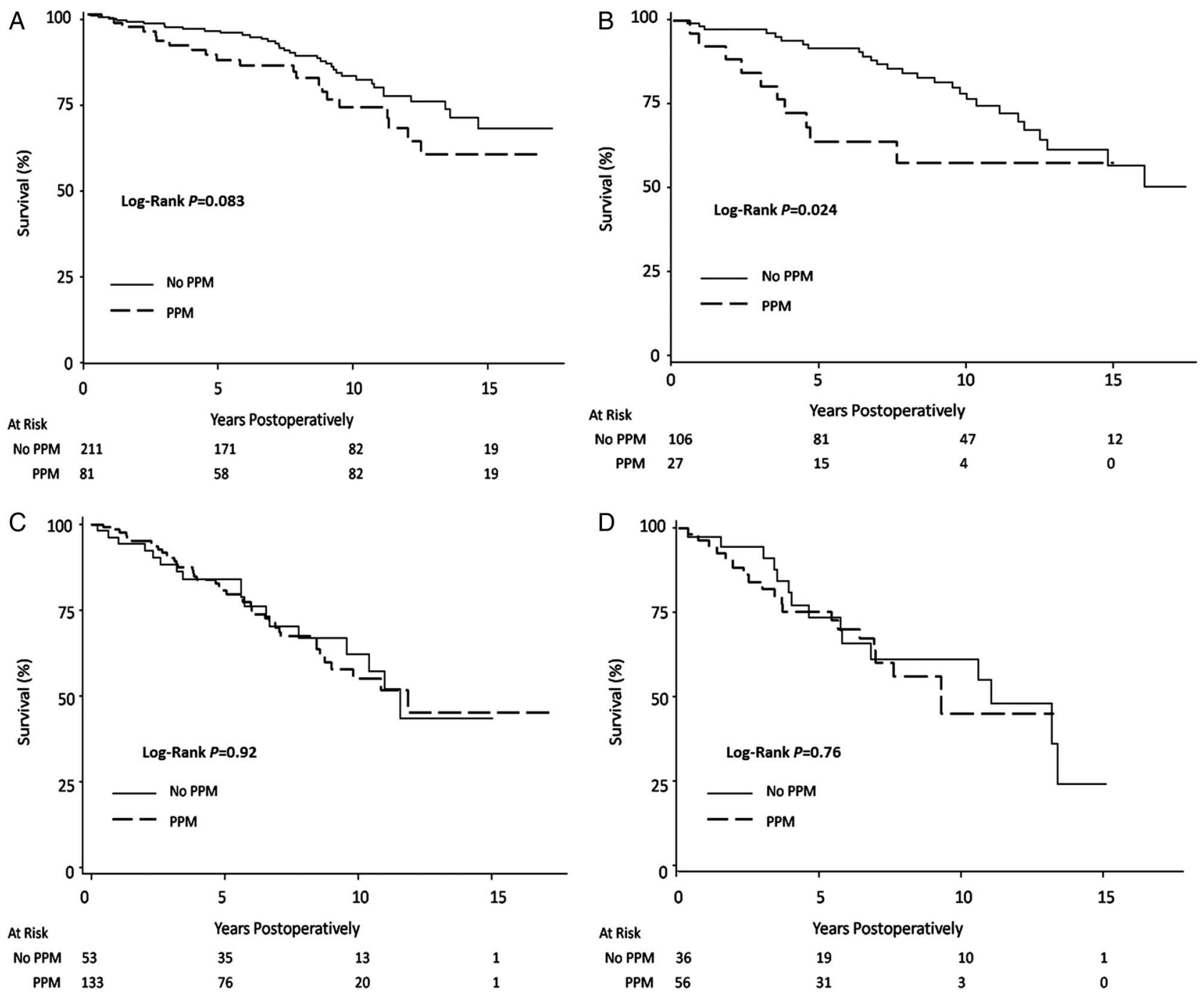

Figure 1 Effect of prosthesis-patient mismatch (PPM) on survival. The effect of PPM on survival after aortic valve replacement in patients (A) under age 70 years with normal left ventricular function, (B) under 70 years of age with left ventricular dysfunction, (C) 70 years old and older with normal left ventricular function, and (D) 70 years old and older with left ventricular dysfunction.

by $28.8 \mathrm{~g} / \mathrm{m}^{2}$ (95\% CI 3.4 to $\left.51.9 ; \mathrm{p}=0.026\right)$. Absolute changes in LV mass were also examined, and similar statistically significant effects were observed (not reported).

To examine the role of PPM severity on consequences of mismatch, a severe PPM threshold was substituted into the models for both subgroups of young patients and elderly patients with normal LV function. No group experienced a significant change in LV mass regression as a result of severe PPM compared to no PPM.

\section{DISCUSSION}

Extensive investigation of PPM has occurred since Rahimtoola initially described this concept. ${ }^{19}$ There is evidence that PPM is associated with poor outcomes in specific subsets of patients. ${ }^{3} 691115$ The inconsistency of published results may be explained by the influence of varying preoperative characteristics and patient comorbidities in study populations and alternative definitions of prosthesis size. Possible modifiers of the effects of PPM include body size, ${ }^{9}{ }^{11}$ LV function ${ }^{6}{ }^{11}$ and, more recently, age at the time of operation. ${ }^{1120}$ Different types of statistical analyses of these interactions may yield different results. The fact that a large series has shown no difference in outcomes with $\mathrm{PPM}^{5}$ may suggest that cohort-specific factors or other risk factors may have stronger effects on survival in certain populations.

Age at the time of operation modifies the relationship between PPM and outcomes after AVR. The presence of PPM, at a threshold of $0.85 \mathrm{~cm}^{2} / \mathrm{m}^{2}$, in patients under age 70 years is independently associated with decreased long-term survival, increased CHF symptoms or CHF-related death and increased combined death or CHF if preoperative LV dysfunction exists. By contrast, there is no adverse clinical effect of PPM in patients less than 70 years of age with normal LV function or in patients over 70 years of age, regardless of LV function. These findings remain true even if PPM is severe.

Patients 70 years old or older have an impaired ability to regress their LV hypertrophy after AVR, and the subset of elderly patients with LV dysfunction suffered reduced LV mass regression as a result of PPM. Taken together, it may be that PPM interacts with age in reducing the physiologic ability of LV mass to regress after AVR, especially if the LV function is already depressed before operation.

We performed several sensitivity analyses to examine the robustness of our conclusions. The results of the primary analyses suggested that age 70 years was a threshold for adverse outcomes in patients with abnormal LV function but not in 

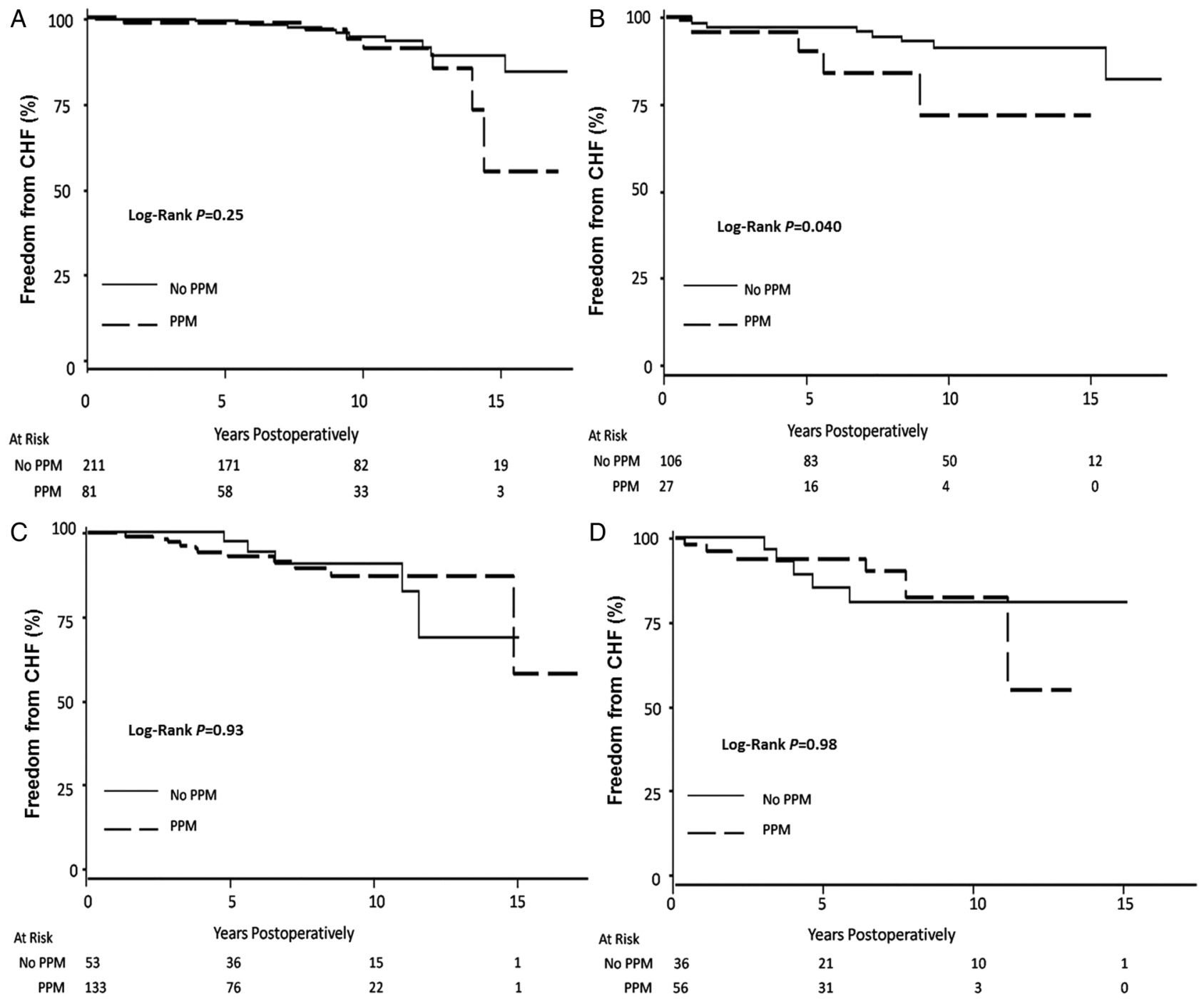

Figure 2 Effect of prosthesis-patient mismatch (PPM) on freedom from congestive heart failure (CHF). The effect of PPM on freedom from CHF recurrence or CHF-related death after aortic valve replacement in patients (A) under age 70 years with normal left ventricular function, (B) under 70 with left ventricular dysfunction, (C) 70 years old and older with normal left ventricular function, and (D) 70 years old and older with left ventricular dysfunction.

those with normal LV function. When age thresholds of 65 years and 60 years were substituted into the Cox models for survival and $\mathrm{CHF}$ in patients with normal LV function, no signal was evident. This analysis supports our choice of age 70 years as an inflection point and our conclusion that in patients with normal LV function, surgical manoeuvres to avoid PPM may be unnecessary, perhaps even in younger patients. It additionally suggests that LV function interacts more strongly with PPM than the age of the patient.

A second set of sensitivity analyses explored the role of PPM severity. Results of the primary analyses suggested that PPM did not reduce outcomes in young patients with normal LV function or in elderly patients. As such, a severe PPM threshold was substituted into the Cox models for these three subgroups. The fact that severe PPM did not result in poorer outcomes may suggest that LV function and age could be more important considerations to outcome than the severity of PPM.

\section{Comparison with previous studies}

We previously demonstrated that the adverse effects of PPM on survival occur primarily in patients with abnormal LV function. ${ }^{6}$
The present analysis demonstrates that PPM resulted in decreased survival and increased CHF specifically in the subset of younger patients with LV dysfunction.

In 2009, Moon et $a l^{20}$ examined their series of 1399 patients who received bioprosthetic AVR. Patients aged 70 years and less experienced impaired long-term survival with PPM $(\mathrm{p}=0.02)$. Patients over 70 years of age experienced no difference in survival with PPM $(\mathrm{p}=0.25)$.

More recently, Mohty et al ${ }^{11}$ examined the effect of PPM in a cohort of over 2500 patients. The authors demonstrated that severe PPM $\left(\leq 0.65 \mathrm{~cm}^{2} / \mathrm{m}^{2}\right)$ is associated with reduced longterm survival in patients less than 70 years old but not older patients. Our findings suggest that this is true only in the subset of young patients with LV dysfunction. Furthermore, the authors demonstrated that moderate PPM is associated with reduced survival in patients with reduced LV function but not preserved LV function. While this is consistent with previous findings from our group, ${ }^{6}$ our current findings suggest that this is only observed in younger patients.

Finally, our results are generally concordant with a 2013 meta-analysis of 14 observational studies, including 14874 

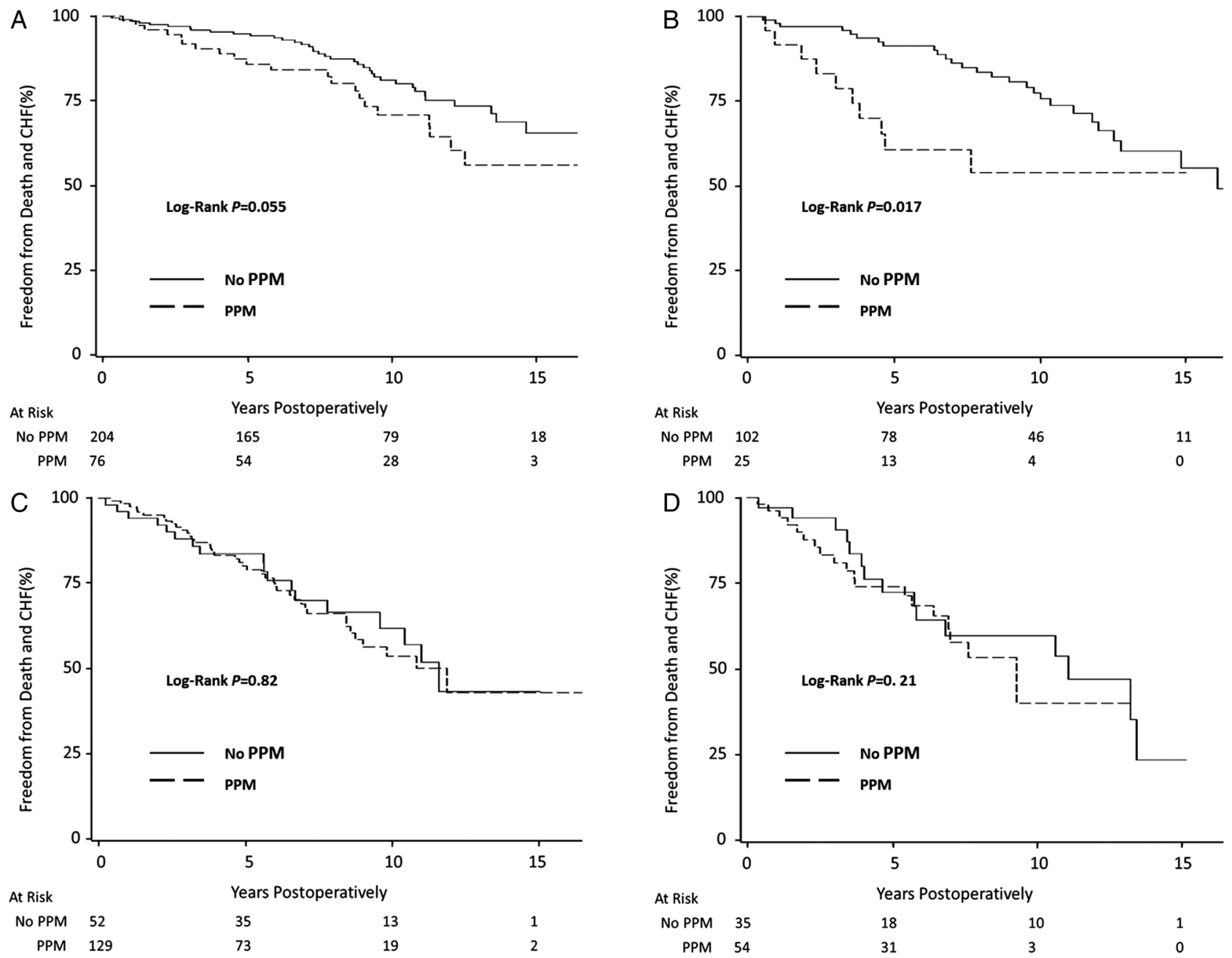

Figure 3 Effect of prosthesis-patient mismatch (PPM) on freedom from death and congestive heart failure (CHF). The effect of PPM on freedom from death and CHF after aortic valve replacement in patients (A) under age 70 with normal left ventricular function, (B) under 70 years of age with left ventricular dysfunction, (C) 70 years old and older with normal left ventricular function, and (D) 70 years old and older with left ventricular dysfunction.

patients, examining the effect of PPM on mortality. ${ }^{21}$ Chen et al reported in subgroup analysis, that PPM was associated with higher mid-term and long-term mortality rates only in younger populations.

\section{Interaction between age and LV function}

The age-specific findings stratified by LV function, as well as the CHF outcomes and LV mass regression are novel, and provide mechanistic correlation with the current survival findings and previously published outcomes. The finding that LV mass regression is reduced in elderly patients with PPM and LV dysfunction has not been previously demonstrated. In patients medicated for hypertension, elderly patients more frequently have residual LV hypertrophy. ${ }^{22}$ In patients receiving AVR, advanced age has been associated with reduced LV mass regression. ${ }^{23}$ It could be that PPM interacts with this reduced physiologic ability to regress LV hypertrophy, explaining the current results. This effect could become significant if an elderly patient had longer life expectancy and was more physically active.

\section{Limitations}

Surgeons tend to avoid surgical manoeuvres to implant larger valves, in elderly patients. This may make it appear that older patients receiving smaller valves have worse outcomes. We have attempted to control for this through the use of stratified analysis and multivariable modelling.

EOA for prosthetic valves, particularly bioprosthetic valves can change over time due to a number of factors. In vivo EOAs were derived from normally functioning valves in prior to the onset of structural valve deterioration.

The number of events in certain subgroups was small. Even though the Cox models were not overfit, the power to draw conclusions was somewhat limited. This is particularly true within the sensitivity analyses of severe PPM. The conclusions drawn from this analysis should be treated as exploratory.

We quantified PPM by using the in vivo EOAs values derived from normally functioning valves examined echocardiographically in healthy patients. We and others have demonstrated that the use of in vivo EOAs, derived in this fashion, correlated well with transprosthesis gradients and postoperative outcomes. ${ }^{2} 316$ Using the EOA derived by the Doppler-echo continuity equation in individual patients after prosthesis implantation has several limitations related to difficulties of accurately measuring the LV outflow diameter caused by reverberations from the prosthetic valve and the presence of large localised transprosthesis gradients and non-flat transprosthetic spatial velocity profiles, which 

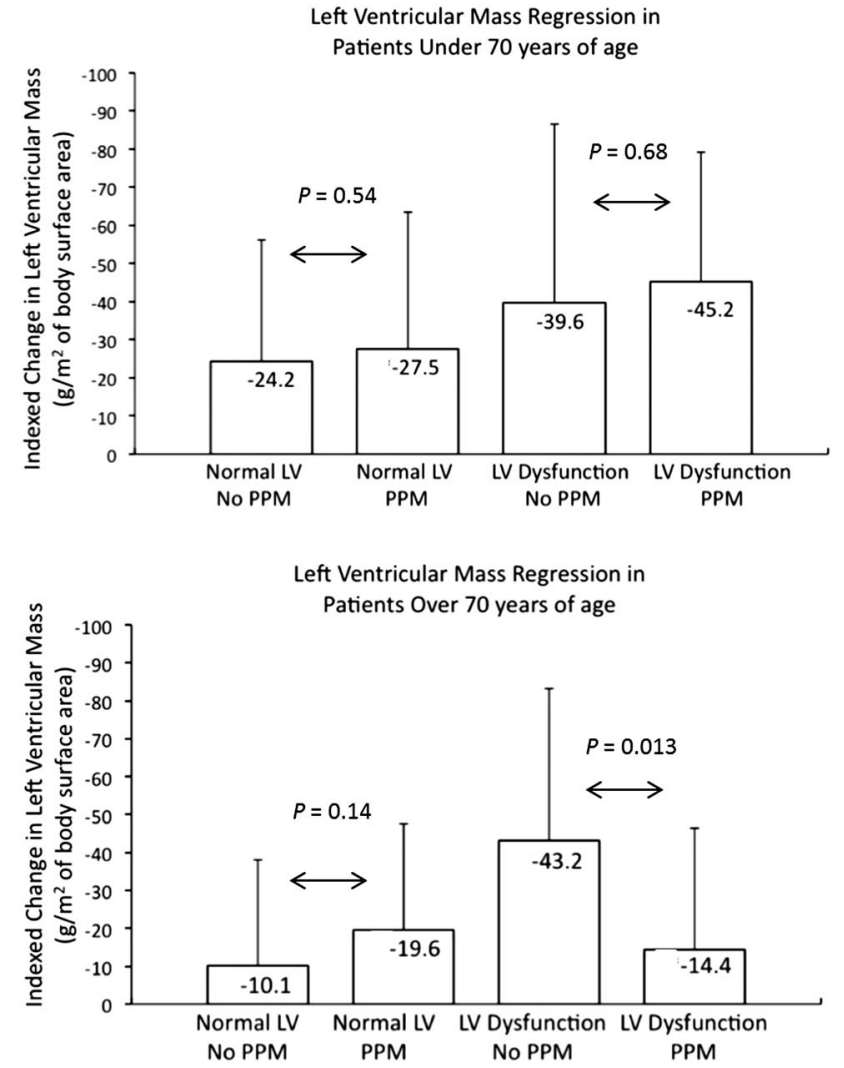

Figure 4 Effect of prosthesis-patient mismatch (PPM) on LV mass. Indexed change in LV mass in patients under age 70 years, and in patients 70 years old and older. In older patients with LV dysfunction, those with PPM experienced a significant reduction in the regression of LV hypertrophy.

frequently result in discrepancies. ${ }^{24-26}$ However, it was demonstrated that the in vivo EOA method provides superior sensitivity and specificity for prediction of postoperative PPM in individual patients. ${ }^{27}$

In clinical practice, PPM, particularly severe PPM, should not be simply dismissed as insignificant in all older patients and those with normal LV function. If severe PPM were to be so extreme as to approximate the EOA of severe AS, the benefits of AVR are clearly lost. Additionally, the number of patients with severe PPM in this study was small and the results should be interpreted cautiously. The findings of this study should be considered along with all other patient factors to determine the appropriate course of action.

\section{CONCLUSIONS}

These results suggest that surgical manoeuvres to increase the in vivo EOA of an implanted valve should be reserved for the subset of patients less than 70 years of age and with LV dysfunction. In patients 70 years old and older, manoeuvres to avoid PPM, which may be associated with increased surgical risk, do not appear justified on the basis of improving postoperative survival or reducing postoperative CHF. However, consideration to avoiding PPM in elderly patients with LV dysfunction may be justified to help avoid impairment of LV mass regression if functional status is good and long-term survival appears favourable.

\section{Key messages}

What is known on this subject?

The impact of prosthesis-patient mismatch (PPM) on outcomes after aortic valve replacement continues to be debated. While some maintain it has no effect on mortality, there is a growing body of evidence that PPM is associated with increased mortality, reduced LV mass regression and increased incidence of postoperative congestive heart failure (CHF). Some published data indicate that the effect of PPM may be modified by factors, such as left ventricular function, Body Mass Index and age.

\section{What might this study add?}

This paper confirms and demonstrates poor long-term survival and decreased freedom from CHF in patients under 70 years old with LV dysfunction. Additionally, PPM appears to reduce LV mass regression in elderly patients with LV dysfunction.

\section{How might this impact on clinical practice?}

The results suggest that surgical manoeuvres to increase the in vivo effective orifice area of the implanted valve should be reserved for the subset of patients less than 70 years of age and with LV dysfunction. In patients 70 years old and older, technical steps in order to avoid PPM, which may be associated with increased surgical risk, do not appear justified on the basis of improving postoperative survival or reducing postoperative CHF.

Acknowledgements We would like to thank Dr Lise M. Bjerre, MD, PhD, for her assistance with the statistical analysis.

Contributors Conception or design of the work: JP, MR, TGM. Acquisition, analysis, or interpretation of data: HT, B-KL, HL. Drafting the work: JP, MR, HT. Revising it critically for important intellectual content: HL, B-KL, TGM. Final approval of the version to be published: JP, HT, B-KL, HL, TGM, MR. Agreement to be accountable for all aspects of the work: JP, MR.

Competing interests MR: Medtronic, Research grant from Edwards Lifesciences. B-KL: Medtronic

Ethics approval Ottawa Heart Institute Research Ethics Board.

Provenance and peer review Not commissioned; externally peer reviewed.

\section{REFERENCES}

1 Bonow RO, Carabello BA, Chatterjee K, et al. ACC/AHA 2006 guidelines for the management of patients with valvular heart disease: a report of the american college of cardiology/american heart association task force on practice guidelines (writing committee to revise the 1998 guidelines for the management of patients with valvular heart disease) developed in collaboration with the society of cardiovascular anesthesiologists endorsed by the society for cardiovascular angiography and interventions and the society of thoracic surgeons. J Am Coll Cardiol 2006;48:e1-148

2 Dumesnil JG, Pibarot P. Prosthesis-patient mismatch and clinical outcomes: The evidence continues to accumulate. J Thorac Cardiovasc Surg 2006;131:952-5.

3 Price J, Lapierre $H$, Ressler L, et al. Prosthesis-patient mismatch is less frequent and more clinically indolent in patients operated for aortic insufficiency. J Thorac Cardiovasc Surg 2009;138:639-45.

4 Howell NJ, Keogh BE, Ray D, et al. Patient-prosthesis mismatch in patients with aortic stenosis undergoing isolated aortic valve replacement does not affect survival. Ann Thorac Surg 2010;89:60-4.

5 Jamieson WR, Ye J, Higgins J, et al. Effect of prosthesis-patient mismatch on long-term survival with aortic valve replacement: assessment to 15 years. Ann Thorac Surg 2010;89:51-8; discussion 59.

6 Ruel M, Al-Faleh H, Kulik A, et al. Prosthesis-patient mismatch after aortic valve replacement predominantly affects patients with preexisting left ventricular dysfunction: Effect on survival, freedom from heart failure, and left ventricular mass regression. J Thorac Cardiovasc Surg 2006;131:1036-44.

7 Blais C, Dumesnil JG, Baillot R, et al. Impact of valve prosthesis-patient mismatch on short-term mortality after aortic valve replacement. Circulation 2003;108:983-8. 
8 Mohty-Echahidi D, Malouf JF, Girard SE, et al. Impact of prosthesis-patient mismatch on long-term survival in patients with small St Jude medical mechanical prostheses in the aortic position. Circulation 2006;113:420-6.

9 Moon MR, Pasque MK, Munfakh NA, et al. Prosthesis-patient mismatch after aortic valve replacement: impact of age and body size on late survival. Ann Thorac Surg 2006;81:481-8.

10 Walther T, Rastan A, Falk V, et al. Patient prosthesis mismatch affects short- and long-term outcomes after aortic valve replacement. Eur J Cardiothorac Surg 2006;30:15-19.

11 Mohty D, Dumesnil JG, Echahidi N, et al. Impact of prosthesis-patient mismatch on long-term survival after aortic valve replacement: influence of age, obesity, and left ventricular dysfunction. J Am Coll Cardiol 2009;53:39-47.

12 Fuster RG, Montero Argudo JA, Albarova OG, et al. Patient-prosthesis mismatch in aortic valve replacement: really tolerable? Eur J Cardiothorac Surg 2005;27:441-9.

13 Tasca $G$, Brunelli F, Cirillo M, et al. Impact of valve prosthesis-patient mismatch on left ventricular mass regression following aortic valve replacement. Ann Thorac Surg 2005;79:505-10.

14 Ruel M, Kulik A, Rubens FD, et al. Late incidence and determinants of reoperation in patients with prosthetic heart valves. Eur J Cardiothorac Surg 2004;25:364-70.

15 Kulik A, Burwash IG, Kapila V, et al. Long-term outcomes after valve replacement for low-gradient aortic stenosis: impact of prosthesis-patient mismatch. Circulation 2006;114(1 Suppl):1553-8.

16 Pibarot $\mathrm{P}$, Dumesnil JG. Hemodynamic and clinical impact of prosthesis-patient mismatch in the aortic valve position and its prevention. J Am Coll Cardiol 2000;36:1131-41.

17 Sahn DJ, DeMaria A, Kisslo J, et al. Recommendations regarding quantitation in M-mode echocardiography: results of a survey of echocardiographic measurements. Circulation 1978;58:1072-83.

18 Devereux RB, Alonso DR, Lutas EM, et al. Echocardiographic assessment of left ventricular hypertrophy: comparison to necropsy findings. Am J Cardiol 1986:57:450-8.
19 Rahimtoola SH. The problem of valve prosthesis-patient mismatch. Circulation 1978;58:20-4.

20 Moon MR, Lawton JS, Moazami N, et al. POINT: prosthesis-patient mismatch does not affect survival for patients greater than 70 years of age undergoing bioprosthetic aortic valve replacement. J Thorac Cardiovasc Surg 2009;137:278-83.

21 Chen J, Lin Y, Kang B, et al. Indexed effective orifice area is a significant predictor of higher mid- and long-term mortality rates following aortic valve replacement in patients with prosthesis-patient mismatch. Eur J Cardiothorac Surg 2014; 45:234-40.

22 Gerdts E, Roman MJ, Palmieri V, et al. Impact of age on left ventricular hypertrophy regression during antihypertensive treatment with losartan or atenolol (the LIFE study). J Hum Hypertens 2004;18:417-22.

23 Cramariuc D, Gerdts E, Segadal L. Impact of hypertension on left ventricular hypertrophy regression and exercise capacity in patients operated for aortic valve stenosis. Scand Cardiovasc J 2006;40:167-74.

24 Vandervoort PM, Greenberg NL, Powell KA, et al. Pressure recovery in bileaflet heart valve prostheses. Localized high velocities and gradients in central and side orifices with implications for doppler-catheter gradient relation in aortic and mitral position. Circulation 1995;92:3464-72.

25 Henneke KH, Pongratz G, Bachmann K. Limitations of doppler echocardiography in the assessment of prosthetic valve hemodynamics. J Heart Valve Dis 1995; 4:18-25

26 Baumgartner $\mathrm{H}$, Khan $\mathrm{S}$, DeRobertis $\mathrm{M}$, et al. Effect of prosthetic aortic valve design on the Doppler-catheter gradient correlation: an in vitro study of normal St. Jude, Medtronic-Hall, Starr-Edwards and Hancock valves. J Am Coll Cardiol 1992; 19:324-32.

27 Bleiziffer S, Eichinger WB, Hettich I, et al. Prediction of valve prosthesis-patient mismatch prior to aortic valve replacement: which is the best method? Heart 2007;93:615-20. 